\title{
Intraoperative test stimulation versus stereotactic accuracy as a surgical end point: a comparison of essential tremor outcomes after ventral intermediate nucleus deep brain stimulation
}

\author{
Tsinsue Chen, MD, ${ }^{1}$ Zaman Mirzadeh, MD, PhD, ${ }^{1}$ Kristina M. Chapple, PhD, ${ }^{1}$ \\ Margaret Lambert, RN, BSN, ${ }^{1}$ Virgilio G. H. Evidente, MD, ${ }^{3}$ Guillermo Moguel-Cobos, MD, ${ }^{2}$ \\ Srivadee Oravivattanakul, MD, ${ }^{2}$ Padma Mahant, MD, ${ }^{2}$ and Francisco A. Ponce, MD ${ }^{1}$ \\ Departments of ${ }^{1}$ Neurosurgery and ${ }^{2}$ Neurology, Barrow Neurological Institute, St. Joseph's Hospital and Medical Center, \\ Phoenix; and ${ }^{3}$ Department of Neurology, Movement Disorders Center of Arizona, Scottsdale, Arizona
}

\begin{abstract}
OBJECTIVE Ventral intermediate nucleus deep brain stimulation (DBS) for essential tremor is traditionally performed with intraoperative test stimulation and conscious sedation, without general anesthesia (GA). Recently, the authors reported retrospective data on 17 patients undergoing DBS after induction of GA with standardized anatomical coordinates on T1-weighted MRI sequences used for indirect targeting. Here, they compare prospectively collected data from essential tremor patients undergoing DBS both with GA and without GA (non-GA).

METHODS Clinical outcomes were prospectively collected at baseline and 3-month follow-up for patients undergoing DBS surgery performed by a single surgeon. Stereotactic, euclidean, and radial errors of lead placement were calculated. Functional (activities of daily living), quality of life (Quality of Life in Essential Tremor [QUEST] questionnaire), and tremor severity outcomes were compared between groups.

RESULTS Fifty-six patients underwent surgery: 16 without GA (24 electrodes) and 40 with GA (66 electrodes). The mean baseline functional scores and QUEST summary indices were not different between groups $(p=0.91$ and $p=$ 0.59 , respectively). Non-GA and GA groups did not differ significantly regarding mean postoperative percentages of functional improvement (non-GA, 47.9\% vs GA, 48.1\%; $p=0.96$ ) or QUEST summary indices (non-GA, 79.9\% vs GA, $74.8 \% ; p=0.50$ ). Accuracy was comparable between groups (mean radial error $0.9 \pm 0.3 \mathrm{~mm}$ for non-GA and $0.9 \pm 0.4$ $\mathrm{mm}$ for $\mathrm{GA}$ patients) $(p=0.75)$. The mean euclidean error was also similar between groups (non-GA, $1.1 \pm 0.6 \mathrm{~mm}$ vs $\mathrm{GA}, 1.2 \pm 0.5 \mathrm{~mm} ; \mathrm{p}=0.92$ ). No patient had an intraoperative complication, and the number of postoperative complications was not different between groups (non-GA, $n=1$ vs $G A, n=10 ; p=0.16$ ).
\end{abstract}

CONCLUSIONS DBS performed with the patient under GA to treat essential tremor is as safe and effective as traditional DBS surgery with intraoperative test stimulation while the patient is under conscious sedation without GA.

https://thejns.org/doi/abs/10.3171/2017.3.JNS162487

KEY WORDS deep brain stimulation; essential tremor; functional outcomes; general anesthesia; intraoperative imaging; quality-of-life outcomes; tremor severity; functional neurosurgery

$\mathrm{D}$ EEP brain stimulation (DBS) of the ventral intermediate nucleus (VIM) is a well-established therapy for the treatment of essential tremor. ${ }^{4,9,22,30}$ The procedure is traditionally performed with patients under conscious sedation, without general anesthesia (GA), with indirect targeting to place leads at consensus coordinates derived from stereotactic anatomical atlases. Leads are re- positioned, if needed, based on intraoperative test stimulation and electrophysiological mapping results..$^{1,5,17}$ During the past decade, DBS has been performed in patients with Parkinson disease while the patients are under GA. Either the globus pallidus internus or subthalamic nucleus is targeted using MRI, which is then fused to an intraoperative MRI or intraoperative CT (iCT) image to determine

ABBREVIATIONS AC-PC = anterior commissure-posterior commissure; $A D L$ = activities of daily living; DBS = deep brain stimulation; ETRS = Essential Tremor Rating Scale; GA = general anesthesia; HOB = head of bed; iCT = intraoperative CT; QUEST = Quality of Life in Essential Tremor; VIM = ventral intermediate nucleus. SUBMITTED September 27, 2016. ACCEPTED March 6, 2017.

INCLUDE WHEN CITING Published online October 13, 2017; DOI: 10.3171/2017.3.JNS162487. 
lead placement accuracy. ${ }^{16,18,19,23,26,27}$ Stereotactic accuracy is thus the primary surgical end point, and leads are repositioned, as needed, based on the degree of stereotactic error. ${ }^{6,15}$

Because the surgical end point of DBS under GA relies primarily on the target's visibility on MRI, DBS for essential tremor is complicated by the fact that the VIM is not discernible on 3-T MRI. Thus, VIM targeting requires so-called indirect methods. ${ }^{2,24,25}$ Our retrospective data for 17 essential tremor patients confirmed the safety and accuracy of indirect VIM targeting under GA without intraoperative test stimulation, with no difference in postoperative functional outcomes compared with traditional lead placement performed with intraoperative test stimulation and the patient under conscious sedation. ${ }^{7}$ This prospective study compared functional, quality-of-life, and tremor severity outcomes in a large cohort of patients with essential tremor undergoing DBS either with or without GA.

\section{Methods \\ Patients}

All patients who underwent VIM DBS with or without GA between March 1, 2014, and February 29, 2016, for essential tremor were included in the study. Exclusion criteria included prior surgery or radiation treatment for essential tremor; however, no patients met either criterion. The decision whether to perform DBS under GA was based on the referring neurologist's agreement and the patient's preference. During preoperative evaluation, patients were counseled extensively on both techniques, with counseling emphasizing that the method utilizing conscious sedation and intraoperative test stimulation is the more accepted method, with well-established literature supporting its efficacy and outcomes. If the patient had preexisting comorbidities, such as obstructive sleep apnea, claustrophobia, or severe anxiety that may have posed a challenge to participation in intraoperative test stimulation or caused concern for higher airway risk during conscious sedation, then surgery performed under GA was recommended. If there were no preoperative medical concerns, the patient made the decision on his or her own, in agreement with his or her referring neurologist. For each patient, a multidisciplinary consensus team determined whether unilateral or bilateral DBS was appropriate, considering the degree of tremor disability and the extent of bilateral contribution. Patients undergoing bilateral surgery were counseled that bilateral VIM DBS is an off-label use and were informed of potential side effects and risks. This study was approved by the institutional review board at St. Joseph's Hospital and Medical Center, Phoenix, Arizona.

\section{Surgical Procedure and Stereotactic Accuracy Assessment}

Detailed surgical procedures have been published previously. In brief, indirect targeting was performed on T1weighted spoiled gradient-recalled acquisition MRI (General Electric) sequences using $X=10.5 \mathrm{~mm}$ lateral to the third ventricular wall, no more than $14 \mathrm{~mm}$ lateral from midline; $\mathrm{Y}=25 \%$ of the anterior commissure-posterior commissure (AC-PC) distance posterior to the midcommissural point; and $\mathrm{Z}=\mathrm{AC}-\mathrm{PC}$ plane.
All leads were placed using frame-based (Leksell) stereotaxy. Bilateral leads were placed during 1 operation, with the left-sided lead placed first. Contact 1 (model 3387 [Medtronic]) was placed to target. Pulse generators were placed during the same operation for both unilateral and bilateral leads. Operating room times reflect the time between patient entry and exit from the operating room, and case times represent the time from skin incision for electrode placement to closure of the pulse generator.

For the GA group, the frame was placed immediately after induction of GA; 1.25 -mm-slice iCT images were obtained on either a CereTom or BodyTom mobile CT scanner (NeuroLogica Corp.). Medtronic FrameLink software was used for iCT coregistration. The head of bed (HOB) was maintained at $0^{\circ}$ throughout the procedure. A postplacement iCT scan was obtained after lead implantation. Stereotactic error was calculated on FrameLink software, using the preoperative trajectory and position of Contact 1. Euclidean error reflects the error vector magnitude between the planned target coordinates and principal contact coordinates. Radial error reflects the degree of deviation from the planned trajectory. If the radial error was $\geq 2$ $\mathrm{mm}$, the lead was repositioned and another iCT scan was obtained to verify accuracy.

For non-GA patients, the $\mathrm{HOB}$ was positioned at $30^{\circ}$ as needed to aid airway patency during intraoperative sedation and to facilitate patient comfort. After lead placement, patients underwent intraoperative test stimulation without microelectrode recording at $0-10 \mathrm{~V}, 90-\mu$ sec pulse width, and $180-\mathrm{Hz}$ frequency. Electrodes were repositioned to address sensory or capsular side effects seen at unacceptably low stimulation parameters or to address a lack of clinical benefit.

\section{Patient Demographics and Clinical Outcomes Assessment}

Patient demographic data included age, sex, and age at onset of essential tremor. Preoperative and 3-month postoperative assessments of functional, quality-of-life, and tremor severity outcomes were prospectively collected. Functional outcomes were assessed using the Bain and Findley Tremor Activities of Daily Living (ADL) selfquestionnaire for patients with tremor, ${ }^{3}$ and quality-of-life outcomes were assessed using the Quality of Life in Essential Tremor (QUEST) questionnaire. ${ }^{28}$ Tremor severity was assessed with tremor amplitude $(\mathrm{cm})$ and frequency $(\mathrm{Hz})$, measured on an iPhone accelerometer (version 2.5, LiftPulse, Lynx Design). Postural tremor was recorded for the treated hand.

\section{Statistical Analysis}

Results are described using descriptive statistics (counts with percentages and means with standard deviations). The X coordinates are presented as absolute values. Independent-sample t-tests were used to compare outcomes, target coordinates, lead error measurements, and procedure times between groups. A power analysis suggested that 2 groups of 11 non-GA and 33 GA patients would achieve $90 \%$ power to detect a statistically significant difference between the Bain and Findley Tremor ADL means of 60 and 50, assuming a standard deviation of 15. PASS 
TABLE 1. Patient demographics

\begin{tabular}{lccc}
\hline \multicolumn{1}{c}{ Variable } & Non-GA $(n=16)$ & $\mathrm{GA}(\mathrm{n}=40)$ & $\mathrm{p}$ Value \\
\hline Mean age, yrs & $61.7 \pm 15.8$ & $67.7 \pm 8.8$ & 0.17 \\
\hline Mean age at onset, yrs & $36.5 \pm 18.2$ & $44.6 \pm 18.7$ & 0.15 \\
\hline Sex & & & 0.09 \\
\hline$\quad$ Male & $6(19.3 \%)$ & $25(80.7 \%)$ & \\
\hline Female & $10(40.0 \%)$ & $15(60.0 \%)$ & \\
\hline Surgery type & & & 0.30 \\
\hline$\quad$ Unilateral & $8(36.4 \%)$ & $14(63.6 \%)$ & \\
\hline$\quad$ Bilateral & $8(23.5 \%)$ & $26(76.5 \%)$ & \\
\hline Mean length of stay, days & $1.3 \pm 0.8$ & $1.5 \pm 0.8$ & 0.44 \\
\hline
\end{tabular}

Data are presented as the mean \pm SD or number and percentage of total patients by demographic and treatment group.

(version 14, NCSS, LLC) was used for the power analysis, and SPSS Statistics for Windows (version 22, IBM Corp.) was used for all other analyses; $p$ values $<0.05$ were considered statistically significant.

\section{Results}

\section{Patient Demographics, Clinical Outcomes, and Tremor Assessment}

VIM DBS was performed in 56 patients; $16(28.6 \%)$ underwent non-GA placement of 24 electrodes, and 40
(71.4\%) underwent GA placement of 66 electrodes (Table 1). The mean age at surgery was $67.7 \pm 8.8$ years for GA patients and $61.7 \pm 15.8$ years for non-GA patients $(\mathrm{p}=$ 0.17 ). The male-to-female ratio and surgery type (unilateral vs bilateral) were not significantly different between groups ( $p=0.09$ and $p=0.30$, respectively). The mean length of stay was similar $(\mathrm{p}=0.44)$ between groups $(\mathrm{GA}$, $1.5 \pm 0.8$ days vs non-GA, $1.3 \pm 0.8$ days).

Three-month follow-up data were available for 11 nonGA patients and 33 GA patients (Table 2). Of the 12 patients without follow-up data, 5 patients did not attend the follow-up appointment because of geographic distance, and 7 patients could not be contacted. The baseline mean ADL score was similar for non-GA and GA groups (59.6 \pm 21.1 and $60.3 \pm 14.9$, respectively; $p=0.91$ ), as was the mean percentage change from baseline $(47.9 \%$ and $48.1 \%$, respectively; $\mathrm{p}=0.96$ ). Similarly, the mean preoperative QUEST summary index for non-GA and GA groups did not differ significantly $(47.2 \pm 20.6$ and $43.8 \pm 16.8$, respectively; $\mathrm{p}=0.59$ ), and the mean percentages of change were similar (79.9\% and $74.8 \%$, respectively; $\mathrm{p}=0.50$ ). The mean preoperative QUEST domain scores (Table 2) were similar between non-GA and GA groups for communication $(\mathrm{p}=0.88)$, work $(\mathrm{p}=0.80)$, hobbies $(\mathrm{p}=0.27)$, physical $(\mathrm{p}=0.62)$, and psychosocial $(\mathrm{p}=0.58)$, and the mean percentage improvement in QUEST domain scores did not differ by domain between the 2 groups $(p \geq 0.06$ ).

Tremor data were available for 17 sides treated in the non-GA group and for 48 sides treated in the GA group

TABLE 2. Functional and QOL outcomes by treatment group*

\begin{tabular}{|c|c|c|c|c|}
\hline Outcome & Preop Score & Postop Score & $\%$ Change & $p$ Value $†$ \\
\hline Bain \& Findley Tremor ADL & & & & 0.96 \\
\hline Non-GA & $59.6 \pm 21.1$ & $28.5 \pm 3.8$ & $47.9 \pm 16.5$ & \\
\hline $\mathrm{GA}$ & $60.3 \pm 14.9$ & $30.9 \pm 10.6$ & $48.1 \pm 14.1$ & \\
\hline QUEST: summary index & & & & 0.50 \\
\hline Non-GA & $47.2 \pm 20.6$ & $7.9 \pm 9.1$ & $79.9 \pm 21.4$ & \\
\hline $\mathrm{GA}$ & $43.8 \pm 16.8$ & $12.1 \pm 12.5$ & $74.8 \pm 21.6$ & \\
\hline QUEST: communication & & & & 0.72 \\
\hline Non-GA & $24.3 \pm 20.1$ & $3.1 \pm 6.9$ & $61.0 \pm 49.1$ & \\
\hline GA & $25.7 \pm 27.3$ & $6.2 \pm 13.2$ & $55.1 \pm 45.4$ & \\
\hline QUEST: work & & & & 0.67 \\
\hline Non-GA & $27.6 \pm 38.7$ & $2.3 \pm 6.6$ & $60.7 \pm 48.6$ & \\
\hline GA & $30.3 \pm 27.8$ & $10.8 \pm 23.0$ & $53.8 \pm 45.7$ & \\
\hline QUEST: hobbies & & & & 0.06 \\
\hline Non-GA & $71.9 \pm 30.1$ & $13.6 \pm 25.5$ & $60.7 \pm 48.6$ & \\
\hline GA & $58.3 \pm 36.2$ & $17.6 \pm 26.3$ & $53.8 \pm 45.7$ & \\
\hline QUEST: physical & & & & 0.82 \\
\hline Non-GA & $70.5 \pm 20.5$ & $10.6 \pm 7.6$ & $82.5 \pm 12.9$ & \\
\hline GA & $66.6 \pm 22.6$ & $15.8 \pm 17.4$ & $74.0 \pm 25.9$ & \\
\hline QUEST: psychosocial & & & & 0.81 \\
\hline Non-GA & $41.6 \pm 19.5$ & $9.9 \pm 11.9$ & $73.8 \pm 27.8$ & \\
\hline GA & $37.8 \pm 19.9$ & $9.8 \pm 13.2$ & $76.0 \pm 26.9$ & \\
\hline
\end{tabular}

* Results are presented for 11 patients in the non-GA treatment group and for 33 patients in the GA treatment group. Means are presented \pm $\mathrm{SD}$.

$\dagger$ Comparison of mean percentage change from preoperative to postoperative scores for non-GA versus GA patient groups. 
TABLE 3. Tremor amplitude and frequency

\begin{tabular}{ccccc}
\hline Variable & Baseline & Postop & \% Change & p Value $^{*}$ \\
\hline Tremor amplitude $(\mathrm{cm})$ & & & & 0.22 \\
\hline Non-GA $(\mathrm{n}=17)$ & $0.59 \pm 1.05$ & $0.04 \pm 0.03$ & $61.9 \pm 33.8$ & \\
\hline $\mathrm{GA}(\mathrm{n}=48)$ & $0.33 \pm 0.51$ & $0.04 \pm 0.04$ & $72.3 \pm 27.8$ & 0.17 \\
\hline Tremor frequency $(\mathrm{Hz})$ & & & & \\
\hline Non-GA $(\mathrm{n}=17)$ & $5.8 \pm 1.1$ & $3.8 \pm 1.1$ & $32.2 \pm 10.1$ & \\
\hline $\mathrm{GA}(\mathrm{n}=48)$ & $5.9 \pm 1.1$ & $4.4 \pm 1.2$ & $25.5 \pm 16.3$ & \\
\hline
\end{tabular}

Values are presented as the mean $\pm \mathrm{SD}$; analysis is per wire.

* Comparison of mean percentage change from preoperative to postoperative score for non-GA versus GA patient groups.

(Table 3). The mean baseline tremor amplitude was 0.59 $\pm 1.05 \mathrm{~cm}$ for non-GA patients and $0.33 \pm 0.51 \mathrm{~cm}$ for GA patients $(p=0.34)$. The mean baseline tremor frequency was $5.8 \pm 1.1 \mathrm{~Hz}$ for non-GA patients and $5.9 \pm 1.1 \mathrm{~Hz}$ for GA patients $(\mathrm{p}=0.67)$. Tremor amplitude improved to a similar degree in non-GA versus GA groups $(61.9 \%$ vs $72.3 \% ; \mathrm{p}=0.22$ ), and improvement in tremor frequency was also similar (32.2\% vs $25.5 \%$; $\mathrm{p}=0.17)$.

\section{Stereotactic Plan}

Non-GA Group

For the 16 patients undergoing DBS without GA, 24 electrodes were placed: 10 right-sided and 14 left-sided leads. Eight patients each underwent unilateral or bilateral placement. The mean AC-PC distance for the 16 patients was $24.5 \pm 1.4 \mathrm{~mm}$, and the mean target location was $\mathrm{X}$ $=13.1 \pm 0.9 \mathrm{~mm}, \mathrm{Y}=-5.8 \pm 0.5 \mathrm{~mm}$, and $\mathrm{Z}=0 \pm 0 \mathrm{~mm}$. Means did not differ for right-sided versus left-sided ACPC distance, target coordinates, euclidean errors, or vector errors ( $p \geq 0.20$; Table 4$)$. The mean case time was $119.6 \pm 41.0$ minutes for unilateral cases and $164.1 \pm 25.6$ minutes for bilateral cases ( $\mathrm{p}=0.02$; Table 5). The mean operating room time was $202.0 \pm 40.0$ minutes for unilateral cases and $261.4 \pm 57.2$ minutes for bilateral cases $(p$ $=0.04$; Table 5).

\section{GA Group}

For the 40 patients undergoing DBS under GA, 66 leads were placed (30 right side and 36 left side); 14 patients underwent unilateral and 26 underwent bilateral placement. The mean AC-PC distance for the 40 patients was 25.4 $\pm 1.6 \mathrm{~mm}$, and the mean target location was $X=13.7 \pm$ $0.7 \mathrm{~mm}, \mathrm{Y}=-6.1 \pm 0.6 \mathrm{~mm}$, and $\mathrm{Z}=0 \pm 0.1 \mathrm{~mm}$. Differences in AC-PC distance, target coordinates, and radial error were not significant for right-sided versus left-sided leads ( $\mathrm{p} \geq 0.07$; Table 4). The mean case time was $133.4 \pm$ 49.3 minutes for unilateral cases and $146.9 \pm 36.0$ minutes for bilateral cases $(p=0.38$; Table 5$)$. The mean operating room time was $119.16 \pm 68.5$ minutes for unilateral cases and $225.4 \pm 55.9$ minutes for bilateral cases $(\mathrm{p}=$ 0.79 ; Table 5).

\section{GA Versus non-GA Groups}

For all right-sided leads, the AC-PC distance was significantly greater for the GA group than for the non-GA group $(25.5 \pm 1.6 \mathrm{~mm}$ vs $24.2 \pm 1.3 \mathrm{~mm}$, respectively; $\mathrm{p}=$ 0.02 ; Table 4). The absolute right-sided $\mathrm{Y}$ value was also significantly larger for the GA group than for the non-GA group $(-6.2 \pm 0.5 \mathrm{~mm}$ vs $-5.6 \pm 0.5 \mathrm{~mm}$, respectively; $\mathrm{p}=$ 0.01 ). The mean coronal angle was also significantly larger in the GA group than in the non-GA group for both rightsided leads $\left(20.0^{\circ} \pm 3.8^{\circ}\right.$ vs $16.8^{\circ} \pm 4.3^{\circ}$, respectively; $\mathrm{p}=$ $0.03)$ and left-sided leads $\left(20.5^{\circ} \pm 2.8^{\circ}\right.$ vs $17.5^{\circ} \pm 4.8^{\circ}$, respectively; $p=0.04$ ). For right-sided leads, the mean third ventricle width was significantly larger for the GA group $(6.5 \pm 2.7 \mathrm{~mm})$ than for the non-GA group $(4.3 \pm 1.4 \mathrm{~mm})$ $(\mathrm{p}=0.02)$. For left-sided leads, the third ventricle width

TABLE 4. DBS target coordinates and lead error measurements

\begin{tabular}{|c|c|c|c|c|c|c|c|c|}
\hline \multirow[b]{2}{*}{ Variable } & \multicolumn{2}{|c|}{ Non-GA } & \multicolumn{2}{|c|}{$\mathrm{GA}$} & \multicolumn{2}{|c|}{ p Value: Rt vs Lt Side } & \multicolumn{2}{|c|}{ p Value: Non-GA vs GA } \\
\hline & Rt Side $(n=10)$ & Lt Side $(n=14)$ & Rt Side $(n=30)$ & Lt Side $(n=36)$ & Non-GA & GA & Rt Side & Lt Side \\
\hline AC-PC (mm) & $24.2 \pm 1.3$ & $24.6 \pm 1.5$ & $25.5 \pm 1.6$ & $25.3 \pm 1.7$ & 0.51 & 0.71 & 0.02 & 0.15 \\
\hline Target $\mathrm{X}(\mathrm{mm})^{*}$ & $13.0 \pm 0.7$ & $13.3 \pm 0.9$ & $13.6 \pm 0.7$ & $13.7 \pm 0.6$ & 0.82 & 0.96 & 0.58 & 0.72 \\
\hline Target $Y(\mathrm{~mm})$ & $-5.6 \pm 0.5$ & $-5.9 \pm 0.5$ & $-6.2 \pm 0.5$ & $-6.1 \pm 0.5$ & 0.20 & 0.58 & 0.01 & 0.25 \\
\hline Target Z (mm) & $0 \pm 0$ & $0 \pm 0$ & $0 \pm 0$ & $0 \pm 0$ & & & & \\
\hline Parasagittal angle $\left({ }^{\circ}\right)$ & $58.1 \pm 4.8$ & $59.0 \pm 6.9$ & $58.4 \pm 4.3$ & $58.5 \pm 4.4$ & 0.93 & 0.96 & 0.82 & 0.79 \\
\hline Coronal angle $\left(^{\circ}\right)$ & $16.8 \pm 4.3$ & $17.5 \pm 4.8$ & $20.0 \pm 3.8$ & $20.5 \pm 2.8$ & 0.72 & 0.53 & 0.03 & 0.04 \\
\hline Euclidean error (mm) & $1.1 \pm 0.8$ & $1.2 \pm 0.3$ & $1.3 \pm 0.5$ & $1.0 \pm 0.5$ & 0.83 & 0.04 & 0.39 & 0.39 \\
\hline Radial error (mm) & $0.8 \pm 0.4$ & $0.9 \pm 0.3$ & $1.0 \pm 0.5$ & $0.8 \pm 0.4$ & 0.64 & 0.07 & 0.30 & 0.47 \\
\hline 3rd ventricle width (mm) & $4.3 \pm 1.4$ & $4.8 \pm 2.0$ & $6.5 \pm 2.7$ & $6.3 \pm 2.6$ & 0.50 & 0.80 & 0.02 & 0.06 \\
\hline
\end{tabular}

Mean \pm SD is presented for target coordinates and error measurements.

* Absolute values. 
TABLE 5. DBS case and operating room times

\begin{tabular}{|c|c|c|c|c|c|}
\hline \multirow[b]{2}{*}{ Time } & \multicolumn{2}{|c|}{ Non-GA } & \multicolumn{2}{|c|}{ GA } & \multirow{2}{*}{$\begin{array}{c}\text { p Value } \\
\text { (non-GA vs GA) }\end{array}$} \\
\hline & Mean \pm SD & No. of Patients & Mean \pm SD & No. of Patients & \\
\hline \multicolumn{6}{|c|}{ Case time (mins) ${ }^{*}$} \\
\hline Unilateral & $119.6 \pm 41.0$ & 8 & $133.4 \pm 49.3$ & 14 & 0.51 \\
\hline Bilateral & $164.1 \pm 25.6$ & 8 & $146.9 \pm 36.0$ & 26 & 0.22 \\
\hline \multicolumn{6}{|c|}{ OR time (mins) $†$} \\
\hline Unilateral & $202.0 \pm 40.0$ & 8 & $219.6 \pm 68.5$ & 14 & 0.53 \\
\hline Bilateral & $261.4 \pm 57.2$ & 8 & $225.4 \pm 55.9$ & 26 & 0.12 \\
\hline
\end{tabular}

was larger for the GA group $(6.3 \pm 2.6 \mathrm{~mm})$ than for the non-GA group $(4.8 \pm 2.0 \mathrm{~mm})(\mathrm{p}=0.06)$. There were no differences in mean case time or mean operating room time for non-GA versus GA groups for either unilateral or bilateral cases ( $\mathrm{p} \geq 0.12$; Table 5).

Non-GA and GA groups did not differ significantly in terms of the overall mean euclidean error $(1.1 \pm 0.6 \mathrm{~mm}$ and $1.2 \pm 0.5 \mathrm{~mm}$, respectively; $\mathrm{p}=0.92$ ) or radial error $(0.9 \pm 0.3 \mathrm{~mm}$ and $0.9 \pm 0.4 \mathrm{~mm}$, respectively; $\mathrm{p}=0.75)$. Within the GA group, euclidean error was significantly higher for right-sided than left-sided leads $(1.3 \pm 0.5 \mathrm{~mm}$ vs $1.0 \pm 0.5 \mathrm{~mm}$, respectively; $p=0.04$ ) (Table 4). However, in a subanalysis of the 52 bilaterally placed leads, right-sided and left-sided errors were not significantly different $(1.4 \pm 0.5 \mathrm{~mm}$ vs $1.1 \pm 0.6 \mathrm{~mm}, \mathrm{p}=0.06)$.

\section{Intraoperative Electrode Repositioning}

The mean number of brain penetrations was $1.1 \pm$ 0.3 both for non-GA patients and for GA patients. One non-GA patient required 2 brain penetrations, and 2 GA patients required 3 penetrations. The non-GA patient underwent bilateral lead placement, and tremor arrest was obtained after the first placement of the right-sided lead; however, due to lack of sensory thresholds, the lead was repositioned posteriorly through a 2-mm offset in attempts to obtain confirmatory sensory side effects. After the lead was repositioned, sensory thresholds at $3.5 \mathrm{~V}$ were obtained, and the electrode was left in place.

One GA patient underwent unilateral left VIM placement, with an initial 2.2-mm medial error. The lead was repositioned using the lateral offset and was found to be $2 \mathrm{~mm}$ lateral. The center trajectory was again used, which placed the lead $1.4 \mathrm{~mm}$ laterally off plan, but within acceptable limits for accuracy. The second GA patient underwent bilateral placement and had a 2.4- $\mathrm{mm}$ posteromedial error on the right. This lead was repositioned $2 \mathrm{~mm}$ anterolaterally; however, the adjustment overcorrected by $2.2 \mathrm{~mm}$. The lead was again placed down the center track, through the second entry point, resulting in a $1.7-\mathrm{mm}$ anterior error.

\section{Stimulator Settings}

Three-month postoperative stimulator settings were available for 14 non-GA patients and 29 GA patients. In
non-GA patients, the most frequent active contacts were Contact 2 for left-sided leads and Contacts 10 and 11 for right-sided leads. In GA patients, the most frequent active contacts were Contact 2 for left-sided leads and Contacts 9 and 11 for right-sided leads. For the GA versus the nonGA groups, findings were similar for the mean amplitude $(2.2 \pm 0.6 \mathrm{~V}$ vs $2.1 \pm 0.7 \mathrm{~V} ; \mathrm{p}=0.54)$, the mean pulse width $(71.7 \pm 15.7$ usec vs $73.2 \pm 19.61$ usec; $p=0.73)$, and the mean frequency $(169.7 \pm 16.5 \mathrm{~Hz}$ vs $171.4 \pm 26.1 \mathrm{~Hz}$; $\mathrm{p}=$ $0.74)$.

\section{Adverse Events}

No patient in either group experienced intraoperative adverse events, defined as any unanticipated complication requiring early procedure termination (e.g., uncontrolled hypertension, seizure, or acute hemorrhage). Postoperative adverse events included procedure-related or hardwarerelated complications occurring up to 3 months postoperatively (Table 6). The total number of adverse events did not differ between non-GA and GA groups (non-GA, $\mathrm{n}=$ 1 vs GA, $n=10 ; p=0.16$ ) or unilateral and bilateral cases $(\mathrm{n}=4$ vs $\mathrm{n}=7 ; \mathrm{p}=0.48)$. The number of patients with stimulation-related side effects did not differ significantly between GA and non-GA patients; however, more patients with stimulation-related side effects had undergone bilateral surgery $(12 / 24,50 \%)$ than unilateral surgery $(6 / 20$, $30 \% ; \mathrm{p}=0.18$ ).

All patients experiencing mental status change postoperatively had resolution of their symptoms prior to discharge. A postoperative hemorrhage occurred in an 82-year-old woman undergoing unilateral lead placement. Nine hours postoperatively, she became confused and moderately aphasic due to a delayed $1.3 \times 1.3 \times 1.5-\mathrm{cm}$ left frontal track hemorrhage. Although clopidogrel had been withheld for 6 days preoperatively, her clopidogrelresponse assay was positive after the hemorrhage was detected. She was discharged home on postoperative Day 3 with a stable hematoma size. At the 3-month follow-up, she had only mild residual aphasia. A wound breakdown occurred in a 60-year-old man, a chronic smoker, who underwent bilateral lead placement under GA. He had 2 episodes of cranial wound breakdown at the same site that required surgical revision. There was no sign of infection after either procedure, and no wound complications were noted at the 16-month follow-up. 
TABLE 6. Adverse events and stimulation-related side effects

\begin{tabular}{|c|c|c|c|c|c|c|}
\hline Variable & Non-GA $(n=11)$ & $\mathrm{GA}(\mathrm{n}=33)$ & $p$ Value & Unilateral $(n=20)$ & Bilateral $(n=24)$ & $\mathrm{p}$ Value \\
\hline \multicolumn{7}{|l|}{ Adverse event } \\
\hline Mental status change & $1(9.1 \%)$ & $8(24.2 \%)$ & 0.28 & $3(15.0 \%)$ & $6(25.0 \%)$ & 0.41 \\
\hline Hemorrhage & $0(0 \%)$ & $1(3.0 \%)$ & 0.56 & $1(5.0 \%)$ & $0(0 \%)$ & 0.27 \\
\hline Wound breakdown & $0(0 \%)$ & $1(3.0 \%)$ & 0.56 & $0(0 \%)$ & $1(4.2 \%)$ & 0.36 \\
\hline Total no. of patients w/ events & $1(9.1 \%)$ & $10(30.3 \%)$ & 0.16 & $4(20.0 \%)$ & $7(29.2 \%)$ & 0.48 \\
\hline \multicolumn{7}{|l|}{ Stimulation-related side effects } \\
\hline Speech disturbance & $3(27.3 \%)$ & $4(12.1 \%)$ & 0.23 & $3(15.0 \%)$ & $4(16.7 \%)$ & 0.88 \\
\hline Balance or gait disturbance & $0(0 \%)$ & $6(18.2 \%)$ & 0.13 & $2(10.0 \%)$ & $4(16.7 \%)$ & 0.52 \\
\hline Speech \& balance disturbances & $2(18.2 \%)$ & $3(9.0 \%)$ & 0.41 & $1(5.0 \%)$ & $4(16.7 \%)$ & 0.23 \\
\hline Total no. of patients w/ side effects & $5(45.5 \%)$ & $13(39.4 \%)$ & 0.72 & $6(30.0 \%)$ & $12(50.0 \%)$ & 0.18 \\
\hline
\end{tabular}

\section{Discussion}

Although DBS performed under conscious sedation with intraoperative test stimulation is the traditionally accepted surgical method, surgery without GA may pose a challenge to a subset of patients with certain medical comorbidities (e.g., obstructive sleep apnea, claustrophobia, anxiety). It is important to note that while only 16 patients in this series elected to undergo surgery without GA, this may not be representative of the actual number of patients who could have medically tolerated surgery without GA, had they not been given a choice between the 2 methods. Some patients without medical limitations may have selected surgery under GA based on preconceived fears or anxieties associated with being awake for a part of the procedure.

While performing surgery under GA using direct targeting of the subthalamic nucleus and globus pallidus internus in Parkinson disease patients has increased in popularity, ${ }_{16,18,19,23,26,27}$ outcomes data are scarce for patients undergoing VIM DBS under GA. Although the subthalamic nucleus and globus pallidus internus are well visualized on MRI sequences, the VIM is not. Thus, VIM DBS performed with the patient under GA must rely on atlas-based or indirect-targeting methods. We hypothesized that, with accurate electrode placement using indirect targeting methods with patients under GA, clinical results would be equivalent to those achieved after leads were placed in traditional DBS surgery without GA.

\section{Clinical Outcomes}

Our initial report of 17 GA patients used retrospective data to demonstrate that the procedure could be performed safely, with submillimeter accuracy $(0.9 \mathrm{~mm}){ }^{7}$ All GA patients had postoperative improvement in their tremor ADL score $(48.6 \%)$, and the degree of improvement did not differ from that of non-GA patients $(45.5 \% ; p=0.35)$. In this prospective study, we found a similar degree of accuracy $(0.9 \mathrm{~mm})$ and tremor ADL improvement $(48.1 \%)$ for the GA group, which was comparable to that of the non-GA group $(47.9 \% ; p=0.96)$. All GA patients had postoperative improvement in QUEST summary index scores (mean change 74.8\%) and all QUEST domain scores. The degree of improvement in the summary index for the GA group was not significantly different from that for the non-GA group $(79.9 \% ; \mathrm{p}=0.50$ ).

Although the Bain and Findley Tremor ADL and QUEST questionnaires are the only 2 assessment tools (of 6 ADL/disability scales and 4 quality-of-life scales) recommended in the recent task force report for evaluation of tremor, 5 rating scales are recommended for evaluating tremor severity. ${ }^{11}$ These scales have good reproducibility; however, they can lose sensitivity at lower and upper ends of tremor amplitude and frequency because of the limited ranges of amplitude and frequency correlated with each score. ${ }^{10,11}$

In an attempt to objectify the tremor measure and reduce interrater error, we used a handheld accelerometer that detects tremor frequency at a range of $0-20 \mathrm{~Hz}$ and at amplitudes of $0-8 \mathrm{~cm}$. Although the use of a mobile telephone-based accelerometer to objectively quantify tremor severity has not been reported previously, the use of other forms of handheld accelerometry and electromyography to measure small changes in tremor amplitude has been described. ${ }^{14,29}$ Wastensson et al ${ }^{29}$ found that measurement of tremor severity using an accelerometer pen correlated strongly with Essential Tremor Rating Scale (ETRS) scores in the stimulation "off" state. However, this agreement was not the case in the stimulation "on" state. They hypothesized that the scale was not as sensitive at discriminating lower-threshold amplitude ranges.

In our study, the degree of postoperative amplitude and frequency improvement did not differ between GA and non-GA groups. We found larger degrees of amplitude improvement in both GA and non-GA groups $(72.3 \%$ and $61.9 \%$, respectively) compared with frequency improvement (25.5\% and $32.2 \%$, respectively). This disparity may be related in part to having measured only postural tremor. Baseline amplitudes and frequencies and degree of postoperative improvement may have been higher if intention tremor had also been measured.

\section{Accuracy and Operating Room Times}

Accuracy in the GA group $(0.9 \mathrm{~mm})$ is similar to that in our previous report ${ }^{7}$ and comparable to previously published results from patients undergoing image-guided DBS 
with GA (0.8-mm radial error using intraoperative MRI ${ }^{19}$ and $1.2-\mathrm{mm}$ radial error using $\mathrm{iCT}) .{ }^{6}$ Our current series demonstrated nearly identical radial errors between nonGA and GA groups, whereas in our initial series, the radial error was higher in the non-GA $(1.3 \mathrm{~mm})$ than in the GA group $(0.9 \mathrm{~mm})(\mathrm{p}=0.06)$. $^{7}$ The standardization of imaging and operating room procedures since our initial report may account for the similarity in radial errors in this study.

In the past, non-GA patients were transported to radiology for preoperative CT scanning after frame placement, while GA patients were maintained with $\mathrm{HOB}$ at $0^{\circ}$ for iCT scanning and the duration of the procedure. With the standardization of non-GA and GA techniques (both groups with frames placed on the operating room table, registration and post-lead placement iCT scans with the patient in the same position), case and operating room times did not differ between our groups. Factors that may confound these data include the fact that all of our patients underwent single-stage lead and battery placement. After leads were placed, the Leksell frame was removed, and the patient was repositioned for pulse generator placement. The time interval between frame removal and setup for the pulse generator placement, including undraping and redraping, may have varied among cases.

\section{Lead Repositioning}

For GA patients, electrodes are repositioned when there is an error $\geq 2 \mathrm{~mm}$, and, for test stimulation patients, electrodes are typically repositioned either because of lack of tremor cessation or unacceptable capsular or sensory side effects at low stimulation thresholds. All patients in this series demonstrated tremor arrest after electrode placement with no side effects at very low thresholds. This finding may be due to the smaller number of non-GA patients in this series, and additional electrodes may have required adjustment had there been a larger number of patients. The 1 repositioned electrode in the non-GA group was moved posteriorly because of a lack of sensory side effects on initial stimulation up to $10 \mathrm{~V}$, which led us to suspect that the lead may have been too anterior despite good tremor control. After its repositioning, sensory thresholds at $3.5 \mathrm{~V}$ were obtained.

\section{Adverse Events}

The adverse events encountered during this study's follow-up period are similar to those seen in previously published DBS series. ${ }^{8,12,21}$ No difference was found between the number of total adverse events in GA versus non-GA groups or between unilateral versus bilateral cases. In the current series, more patients undergoing bilateral thalamic DBS experienced stimulation-related side effects when compared with patients receiving unilateral thalamic DBS, which is in line with what has been previously established in the literature. . $^{4,13,20}$

\section{Limitations}

One of the most significant limitations of this study is the lack of an objective tremor rating scale evaluation (i.e., ETRS). We have since modified our protocol and now con- sistently document these scores for all patients both preoperatively and postoperatively. Measurements of resting, postural, and intention tremor on a handheld accelerometer will be recorded for a future validation study using the ETRS. Assessments were performed by a nonblinded movement disorders specialist nurse, and thus were subject to observer bias. In addition, given that the study was a nonrandomized comparison of 2 surgical techniques selected based on patient and neurologist preference, preconceived notions of anticipated outcomes may have influenced postoperative responses to the QUEST and Bain and Findley questionnaires. The "objective" measure of tremor severity was postural tremor amplitude and frequency as measured by a handheld accelerometer. Resting, kinetic, and intention tremor were not evaluated, nor were measurements of lower-limb, trunk, head, tongue, and voice tremor. Although a handheld accelerometer may theoretically provide more objective measurements of amplitude and frequency, particularly for lower amplitude changes, it cannot be readily applied to the measurement of lowerlimb or axial tremor. Furthermore, mobile measurement applications have not been validated.

Additional limitations of this study include the number of patients lost to follow-up (12 of 56), and the relatively short follow-up period of 3 months. Patients may have opted out of their 3-month follow-up appointment, given that it was not performed in their programming neurologist's office and was scheduled for research purposes only. Reasons for not returning may have included personal inconvenience or geographic distance, especially as some patients in Arizona live out of the state for various parts of the year. Because we are a tertiary referral center, some patients come from out of state for surgery and return home to their local neurologist for programming and continued follow-up. Appointment letters and reminder telephone calls were made in an attempt to obtain clinical follow-up, and 5 of the 12 patients confirmed geographic distance as the reason for not returning; unfortunately, 7 patients could not be contacted. Longer-term follow-up is also needed to assess potential changes in clinical efficacy and stimulation parameters over time, as well as to capture any long-term complications that occur after the 3-month postoperative window.

We noted a convergence in non-GA and GA surgical techniques over time (e.g., standard operating room, Leksell stereotactic frame, iCT, flat bed), such that differences in previously reported errors between techniques were not observed. Regardless of surgical technique, stereotactic error was never more than $2 \mathrm{~mm}$ in the non-GA group, and, by definition, always less than $2 \mathrm{~mm}$ in the GA group. This may confound the difference, or lack thereof, that we report between non-GA and GA patients because an assumption on the part of surgery with test stimulation is that stereotactic error is a moot point if the surgeon-selected "indirect" target was suboptimal and was subsequently corrected using intraoperative mapping.

Ultimately, a randomized trial comparing non-GA and GA postoperative outcomes would be ideal; however, the inherent challenge with this type of trial is the preconceived bias that patients have about surgical techniques that leads them to select one procedure over another. In 
an attempt to obtain higher-quality data to compare techniques, we designed a randomized trial between the nonGA and GA approaches, but after failing to enroll patients for 6 months, we closed the trial (Functional Outcomes of Awake vs. Asleep Deep Brain Stimulation [DBS] for Essential Tremor; clinicaltrials.gov registration no. NCT02418858). Most patients were unwilling to be randomized to a certain technique after they were informed about the risks and benefits of both techniques. Furthermore, we recognize that a single-center randomized study would be plagued by bias such as the observed convergence of technique, and conclusions drawn from such data would be suspect.

Despite these challenges, this study provides a fairly strong comparison between the clinical outcomes of VIM DBS with and without GA, given that all procedures were performed by a single surgeon at a single institution, with uniform surgical techniques and standardized prospectively collected clinical outcomes.

\section{Conclusions}

VIM DBS for essential tremor under GA can be performed safely, with equivalent functional, quality-of-life, and tremor severity outcomes compared with VIM DBS with intraoperative test stimulation without GA.

\section{Acknowledgments}

We thank Loraine Escalante for her assistance with patient care coordination.

Margaret Lambert and Dr. Kristina Chapple receive financial funding from the Barrow Center for Neuromodulation.

\section{References}

1. Alterman RL, Weisz D: Microelectrode recording during deep brain stimulation and ablative procedures. Mov Disord 27:1347-1349, 2012

2. Anthofer J, Steib K, Fellner C, Lange M, Brawanski A, Schlaier J: The variability of atlas-based targets in relation to surrounding major fibre tracts in thalamic deep brain stimulation. Acta Neurochir (Wien) 156:1497-1504, 2014

3. Bain PG, Findley LJ, Atchison P, Behari M, Vidailhet M, Gresty M, et al: Assessing tremor severity. J Neurol Neurosurg Psychiatry 56:868-873, 1993

4. Baizabal-Carvallo JF, Kagnoff MN, Jimenez-Shahed J, Fekete R, Jankovic J: The safety and efficacy of thalamic deep brain stimulation in essential tremor: 10 years and beyond. J Neurol Neurosurg Psychiatry 85:567-572, 2014

5. Benabid AL, Chabardes S, Mitrofanis J, Pollak P: Deep brain stimulation of the subthalamic nucleus for the treatment of Parkinson's disease. Lancet Neurol 8:67-81, 2009

6. Burchiel KJ, McCartney S, Lee A, Raslan AM: Accuracy of deep brain stimulation electrode placement using intraoperative computed tomography without microelectrode recording. J Neurosurg 119:301-306, 2013

7. Chen T, Mirzadeh Z, Chapple K, Lambert M, Dhall R, Ponce FA: "Asleep" deep brain stimulation for essential tremor. J Neurosurg 124:1842-1849, 2016

8. Chen T, Mirzadeh Z, Chapple K, Lambert M, Ponce FA: Complication rates, lengths of stay, and readmission rates in "awake" and "asleep" deep brain simulation. J Neurosurg 127:360-369, 2017
9. Chopra A, Klassen BT, Stead M: Current clinical application of deep-brain stimulation for essential tremor. Neuropsychiatr Dis Treat 9:1859-1865, 2013

10. Elbe RJ, Koller WC: Tremor. Baltimore: John Hopkins University Press, 1990

11. Elble R, Bain P, Forjaz MJ, Haubenberger D, Testa C, Goetz CG, et al: Task force report: scales for screening and evaluating tremor: critique and recommendations. Mov Disord 28:1793-1800, 2013

12. Fenoy AJ, Simpson RK Jr: Risks of common complications in deep brain stimulation surgery: management and avoidance. J Neurosurg 120:132-139, 2014

13. Hwynn N, Hass CJ, Zeilman P, Romrell J, Dai Y, Wu SS, et al: Steady or not following thalamic deep brain stimulation for essential tremor. J Neurol 258:1643-1648, 2011

14. Mansur PH, Cury LK, Andrade AO, Pereira AA, Miotto GA, Soares AB, et al: A review on techniques for tremor recording and quantification. Crit Rev Biomed Eng 35:343-362, 2007

15. Mirzadeh Z, Chapple K, Lambert M, Dhall R, Ponce FA: Validation of CT-MRI fusion for intraoperative assessment of stereotactic accuracy in DBS surgery. Mov Disord 29:17881795,2014

16. Mirzadeh Z, Chapple K, Lambert M, Evidente VG, Mahant P, Ospina MC, et al: Parkinson's disease outcomes after intraoperative CT-guided "asleep" deep brain stimulation in the globus pallidus internus. J Neurosurg 124:902-907, 2016

17. Montgomery EB Jr: Microelectrode targeting of the subthalamic nucleus for deep brain stimulation surgery. Mov Disord 27:1387-1391, 2012

18. Nakajima T, Zrinzo L, Foltynie T, Olmos IA, Taylor C, Hariz MI, et al: MRI-guided subthalamic nucleus deep brain stimulation without microelectrode recording: can we dispense with surgery under local anaesthesia? Stereotact Funct Neurosurg 89:318-325, 2011

19. Ostrem JL, Galifianakis NB, Markun LC, Grace JK, Martin AJ, Starr PA, et al: Clinical outcomes of PD patients having bilateral STN DBS using high-field interventional MR-imaging for lead placement. Clin Neurol Neurosurg 115:708-712, 2013

20. Pahwa R, Lyons KE, Wilkinson SB, Simpson RK Jr, Ondo WG, Tarsy D, et al: Long-term evaluation of deep brain stimulation of the thalamus. J Neurosurg 104:506-512, 2006

21. Patel DM, Walker HC, Brooks R, Omar N, Ditty B, Guthrie BL: Adverse events associated with deep brain stimulation for movement disorders: analysis of 510 consecutive cases. Neurosurgery 11 (Suppl 2):190-199, 2015

22. Picillo M, Fasano A: Recent advances in essential tremor: Surgical treatment. Parkinsonism Relat Disord 22 (Suppl 1):S171-S175, 2016

23. Saleh S, Swanson KI, Lake WB, Sillay KA: Awake neurophysiologically guided versus asleep MRI-guided STN DBS for Parkinson disease: a comparison of outcomes using levodopa equivalents. Stereotact Funct Neurosurg 93:419426, 2015

24. Schaltenbrand G, Wahren W: Atlas for Stereotaxy of the Human Brain, ed 2. Stuttgart: Thieme, 1977

25. Schlaier J, Anthofer J, Steib K, Fellner C, Rothenfusser E, Brawanski A, et al: Deep brain stimulation for essential tremor: targeting the dentato-rubro-thalamic tract? Neuromodulation 18:105-112, 2015

26. Sidiropoulos C, Rammo R, Merker B, Mahajan A, LeWitt P, Kaminski P, et al: Intraoperative MRI for deep brain stimulation lead placement in Parkinson's disease: 1 year motor and neuropsychological outcomes. J Neurol 263:1226-1231, 2016

27. Starr PA, Martin AJ, Ostrem JL, Talke P, Levesque N, Larson PS: Subthalamic nucleus deep brain stimulator placement using high-field interventional magnetic resonance imaging 
and a skull-mounted aiming device: technique and application accuracy. J Neurosurg 112:479-490, 2010

28. Tröster AI, Pahwa R, Fields JA, Tanner CM, Lyons KE: Quality of life in Essential Tremor Questionnaire (QUEST): development and initial validation. Parkinsonism Relat Disord 11:367-373, 2005

29. Wastensson G, Holmberg B, Johnels B, Barregard L: Quantitative methods for evaluating the efficacy of thalamic deep brain stimulation in patients with essential tremor. Tremor Other Hyperkinet Mov (N Y) 3:3, 2013

30. Zhang K, Bhatia S, Oh MY, Cohen D, Angle C, Whiting D: Long-term results of thalamic deep brain stimulation for essential tremor. J Neurosurg 112:1271-1276, 2010

\section{Disclosures}

The authors report no conflict of interest concerning the materi- als or methods used in this study or the findings specified in this paper.

\section{Author Contributions}

Conception and design: Ponce, Chen. Acquisition of data: Chen, Lambert, Evidente, Moguel-Cobos, Oravivattanakul, Mahant. Analysis and interpretation of data: Ponce, Chen, Mirzadeh, Chapple. Drafting the article: Ponce, Chen, Mirzadeh, Chapple. Critically revising the article: all authors. Reviewed submitted version of manuscript: Ponce, Chen. Statistical analysis: Chen, Chapple. Administrative/technical/material support: Lambert. Study supervision: Ponce.

\section{Correspondence}

Francisco A. Ponce, c/o Neuroscience Publications, Barrow Neurological Institute, St. Joseph's Hospital and Medical Center, 350 W Thomas Rd., Phoenix, AZ 85013. email: neuropub@ barrowneuro.org. 